\title{
Resonance fluorescence of a cold atom in a high-finesse resonator
}

\author{
Marc Bienert, ${ }^{1}$ J. Mauricio Torres, ${ }^{1}$ Stefano Zippilli, ${ }^{2,3}$ and Giovanna Morigi ${ }^{2}$ \\ ${ }^{1}$ Instituto de Ciencias Físicas, Universidad Nacional Autónoma de México, 62251 Cuernavaca, Morelos, Mexico \\ ${ }^{2}$ Grup d'Optica, Departament de Fisica, Universitat Autónoma de Barcelona, 08193 Bellaterra, Spain \\ ${ }^{3}$ ICFO_Institut de Ciències Fotòniques, 08860 Castelldefels (Barcelona), Spain
}

(Received 6 February 2007; published 19 July 2007)

\begin{abstract}
We study the spectra of emission of a system composed by an atom, tightly confined inside a high-finesse resonator, when the atom is driven by a laser and is at steady state of the cooling dynamics induced by laser and cavity fields. In general, the spectrum of resonance fluorescence and the spectrum at the cavity output contain complementary information about the dynamics undergone by the system. In certain parameter regimes, quantum interference effects between the scattering processes induced by cavity and laser fields lead to the selective suppression of features of the resonance fluorescence spectrum, which are otherwise visible in the spectrum of laser-cooled atoms in free space.
\end{abstract}

DOI: 10.1103/PhysRevA.76.013410

PACS number(s): 32.80.Pj, 42.50.Vk

\section{INTRODUCTION}

The modification of atomic dynamics by means of optical resonators is actively investigated by several groups worldwide. Several studies have recently focused on the manipulation and control of the atomic center-of-mass motion by mechanical forces induced by the resonator. Recent experiments demonstrated mechanical forces of single photons on single atoms [1-4], cooperative effects and self-organization of laser cooled atomic clouds in resonators [5-7], and cavityassisted cooling of atoms $[8,9]$. The atomic motion and resonator field states are interdependent, as the atomic motion is determined by the mechanical forces of the resonator's field, while the resonator's field is strongly affected by the medium's refractive index and thus by the atom's position and velocity [10]. Information about the atomic motion can be obtained through the emitted light $[4,11]$, and has been used to implement feedback-induced trapping and cooling of the atomic motion $[12,13]$. In general, the quantum behavior of atoms in resonators is still largely unexplored.

In this paper we study theoretically the spectrum of resonance fluorescence and the spectrum at the cavity output of the light scattered by an atom, tightly confined inside an optical resonator and continuously driven by a laser. The photons collected at the detectors are scattered by the atom at the steady state of cavity-assisted cooling and carry the information of the coupled dynamics between cavity and atomic external quantum degrees of freedom. We analyze the spectra in various cavity cooling regimes, which have been identified in [14,15], applying and extending the method in $[16,17]$, used for evaluating the spectrum of resonance fluorescence of a laser cooled atom, to our case, in which the atom is strongly coupled to a mode of the quantum electromagnetic field. This work extends and complements previous theoretical investigations on the property of the light scattered by atoms inside resonators, see, for instance, [18-26], by considering systematically the coupled dynamics between field and quantized atomic motion, which determines the steady state and the properties of the scattered light. Differing from studies on the coupled dynamics of atoms in resonator, where both confinement and cooling are provided by the cavity field potential $[27,28]$, in this work the atom is confined by an external potential, like an ion or a dipole trap, and its motion is cooled by the mechanical effects of laser and cavity mode fields.

This work is organized as follows. In Sec. II the setup of the system is discussed. In Sec. III the theoretical model is introduced, and in Sec. IV the spectra of emission are reported and discussed. Finally, in Sec. V the conclusions and outlooks are presented.

\section{SPECTRA OF EMISSION}

We consider an atom, whose dipole transition couples to the mode of a high-finesse resonator and is driven by a laser, in the setup sketched in Fig. 1. The atomic motion is confined by a tight harmonic potential and is kept cool by the mechanical effects of cavity and laser fields. Two detectors record the photons scattered by the system, which is at the

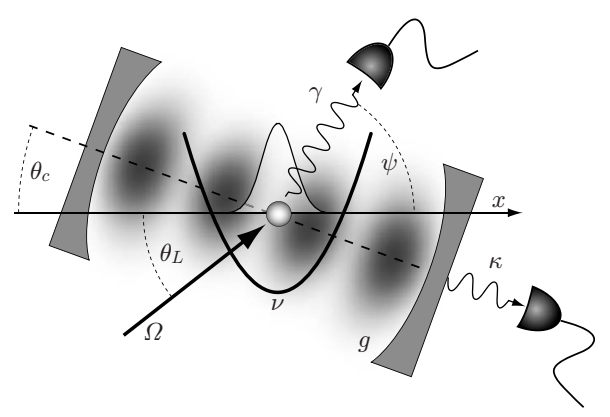

FIG. 1. An atom is confined by an external potential inside an optical resonator. The motion is essentially along the $\hat{x}$ direction (the radial potential is assumed to be very steep) and is harmonic at frequency $\nu$. A mode of the resonator couples with strength $g$ to the dipole, which is driven transversally by a laser at Rabi frequency $\Omega$. The system dissipates by spontaneous emission of the atomic excited state at rate $\gamma$ and by cavity decay at rate $\kappa$. Two detectors measure, respectively, the field at the cavity output and the atomic fluorescence at an angle $\psi$ with respect to the axis of the motion. $\theta_{c}$ and $\theta_{L}$ indicate the angles of the cavity and laser wave vectors with the motional axis. 
stationary state of the cooling process: One detector reveals the spectrum of the intensity of the spontaneously scattered photons, another detector measures the power spectrum of the photons at the cavity output.

The corresponding signals are, apart from a constant proportionality factor, the Fourier transform of the autocorrelation function of the electric field, and take the form

$$
\mathbf{S}^{(j)}(\omega) \propto \operatorname{Re} \int_{0}^{\infty} d \tau e^{-i \omega \tau}\left\langle E_{j}^{(+)}(t+\tau) E_{j}^{(-)}(t)\right\rangle,
$$

where $E^{(+)}(t)$ and $E^{(-)}(t)$ are the negative and positive frequency component of the electric field at the detector $j$ at time $t$, and $\langle\cdot\rangle$ describes the average over the electromagnetic field, the atomic dipole, and center-of-mass degrees of freedom. From now on we label with the superscript " $j=a t$ " the signal of resonance fluorescence and with " $j=$ cav" the signal at the cavity output. In the vacuum the intensity of the scattered field is proportional to the source field. Hence in the far-field of the dipole source the electric field is proportional to the retarded value of the atomic dipole, $E_{\mathrm{at}}^{(-)}(t) \propto D\left(t-t^{\prime}\right)$, with $D$ the dipole operator, and at the cavity output is proportional to the cavity field, $E_{\mathrm{cav}}^{(-)}(t) \propto a\left(t-t^{\prime}\right)$, with $a$ the annihilation operator of a photon of the cavity mode. The power spectra at steady state are defined as

$$
\mathbf{S}^{(j)}(\omega)=\chi^{(j)} \mathcal{S}^{(j)}(\omega),
$$

where $\chi^{\text {(at) }}$ and $\chi^{\text {(cav) }}$ are prefactors which scale with the spontaneous decay rate $\gamma$ and the cavity decay $\kappa$, respectively. In the reference frame of the laser at frequency $\omega_{L}$, the spectral form is given by

$$
\begin{aligned}
& \mathcal{S}^{(\mathrm{at})}(\omega)=\operatorname{Re} \int_{0}^{\infty} d \tau e^{-\mathrm{i}\left(\omega-\omega_{L}\right) \tau}\left\langle D^{\dagger}(\tau) D(0)\right\rangle_{\mathrm{st}}, \\
& \mathcal{S}^{(\mathrm{cav})}(\omega)=\operatorname{Re} \int_{0}^{\infty} d \tau e^{-\mathrm{i}\left(\omega-\omega_{L}\right) \tau}\left\langle a^{\dagger}(\tau) a(0)\right\rangle_{\mathrm{st}},
\end{aligned}
$$

where the average $\langle\cdot\rangle$ is evaluated by tracing over the atomic and cavity degrees of freedom, which are treated quantum mechanically. In this work we systematically consider the coupling of the cavity field with the quantized center-of-mass motion in determining the cooling dynamics and steady state, and its manifestations in the transmission spectrum and in the spectrum of resonance fluorescence. In particular, we focus on the peculiar properties which are due to the presence of the resonator. For this purpose, we shall give a brief review of some basic properties of the spectrum of a trapped and laser-cooled atomic dipole in free space.

The processes leading to cooling of an atom confined by a harmonic trap and in free space give rise to sidebands of the peaks of the spectrum of resonance fluorescence. They result from the mechanical processes associated with the change of the motional state by one excitation. The most evident features are the sidebands of the elastic peak, which contain the most information on the cooling process [29-31]. In particular, for a two-level atom cooled by a laser at frequency $\omega_{L}$, the lower sideband of the elastic peak, at frequency $\omega_{L}-\nu$, corresponds to photons which heat the motion of one excita- tion, while the upper sideband, at $\omega_{L}+\nu$, to photons which cool the motion. Cooling is achieved by enhancing the rate of scattering of cooling photons over the heating photons. At steady state the number of heating and cooling photons per unit time is equal and, below saturation, the two sideband signals integrated over all the solid angle of emission are Lorentzian curves of the same height and width [29]. Nevertheless, at a specific detection (emission) angle, the sidebands of the elastic peak are asymmetric in height and in their functional dependence on the frequency. This behavior is due to quantum interference between paths of excitations coupling internal and external degrees of freedom [30]. In [17] it has been shown that a similar behavior can be observed for the sidebands of the inelastic spectrum.

A high-finesse resonator modifies significantly the scattering properties of the atom. In the regime of strong coupling the resonator saturates the dipole transition at the single photon level [24]. This property gives rise to inelastic features of the resonance fluorescence spectrum, which are already visible when the dipole is driven by a weak laser [25]. When additionally the atomic motion is considered, the presence of an optical resonator can give rise to a rich variety of interference effects, which, among others, affect the features of the motional sidebands. In the limit of weak drives the relevant scattering processes which cool and heat the atomic motion are depicted in Figs. 2 and 3 [15]. In the figures, the levels coupled by the laser are the stable state $\left|g, 0_{c}, n\right\rangle$ and the dressed states $| \pm, n\rangle$, which are the eigenstates of dipolecavity interaction and are superpositions of the states $\left|g, 1_{c}, n\right\rangle$ and $\left|e, 0_{c}, n\right\rangle$. Here, $|g\rangle$ and $|e\rangle$ are the electronic ground and excited states of the dipole transition, $\left|0_{c}\right\rangle$ and $\left|1_{c}\right\rangle$ are the cavity mode state with 0 and 1 photons, and $|n\rangle$ denotes the vibrational excitations. Figure 2 describes the contributions which add up coherently when the atom is scattered in a different motional state, $n \rightarrow n \pm 1$, and the photon is dissipated by atomic spontaneous emission. In Fig. 2(a) the driving laser, at Rabi frequency $\Omega$, excites the system without modifying the motional state which, instead, is changed by the recoil induced by the spontaneously emitted photon. Here the mechanical effects are scaled by the geometric factor $\alpha$ which is found after averaging over the solid angle of spontaneous emission, see Sec. III A. In Fig. 2(b) the change in the center-of-mass state is due to the mechanical coupling with the laser and in Fig. 2(c) with the cavity field at vacuum Rabi frequency $\tilde{g}$. The parameters $\varphi_{L}$ and $\varphi_{c}$, which are defined in Sec. III A, depend on the geometry of the setup and scale the recoil effect of a laser photon and of a cavity photon, respectively. Figure 3 describes the corresponding processes when the photon is dissipated by cavity decay. In [15] quantum interference between the processes depicted in Fig. 2 has been used in order to reduce the ratio $A_{+} / A_{-}$between the rates of heating and of cooling, and therefore to enhance the cooling efficiency. We remark that a transition $\left|g, 0_{c}, n\right\rangle \rightarrow\left|g, 0_{c}, n \pm 1\right\rangle$ corresponds to the emission of a photon at frequency $\omega_{L} \mp \nu$, and to a corresponding signal in the spectrum of emission. Interference between the scattering processes, leading to photon emission, will give a corresponding modification of the sideband signals, which will be generally different from the one observed for sideband 

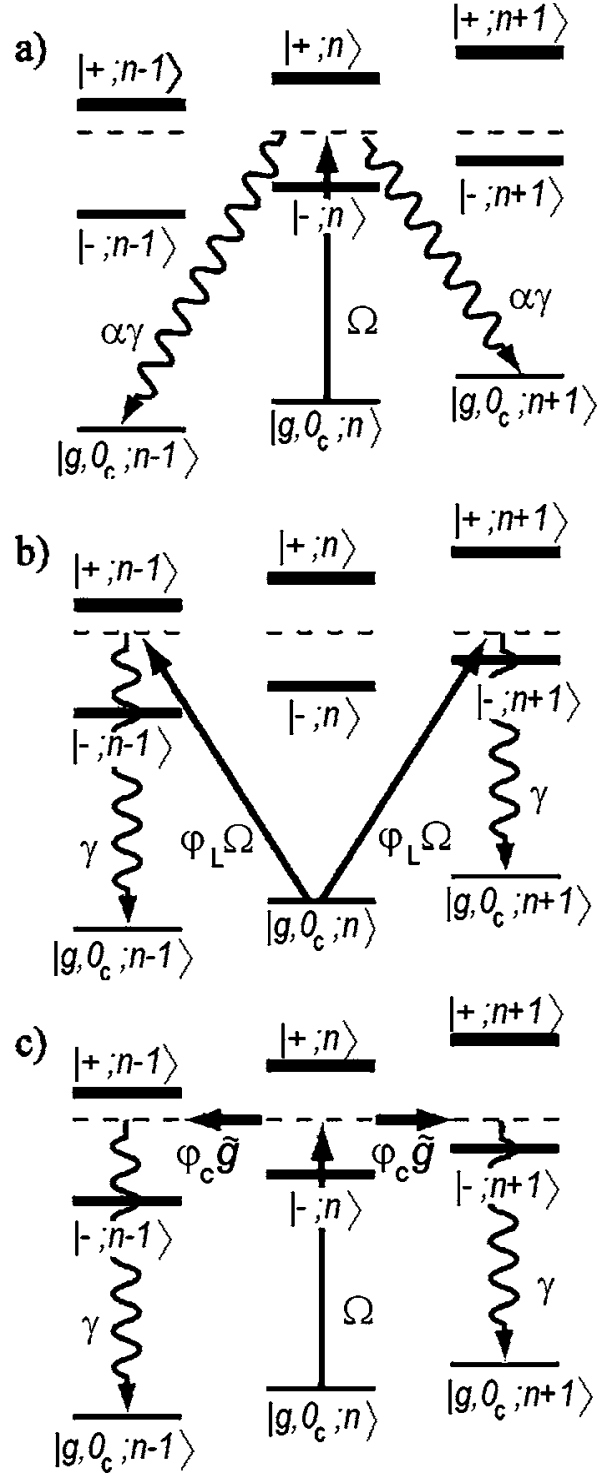

FIG. 2. Scattering processes, where the photon is emitted by spontaneous emission, leading to a change of the vibrational number. The states $\left|g, 0_{c} ; n\right\rangle,| \pm ; n\rangle$ are the cavity-atom dressed states at phonon number $n$. The parameters $\alpha, \varphi_{c}$, and $\varphi_{L}$ emerge from the mechanical effects of light and are defined in Sec. III A.

cooling in free space. We refer the reader to Sec. IV, where the evaluated spectra are shown and discussed.

\section{MODEL}

The spectra of emission in Eqs. (3) and (4) are evaluated by averaging over the atomic and cavity degrees of freedom, namely tracing over the density matrix $\varrho$ for the cavity mode, dipole, and quantized center-of-mass motion. The two-time correlation functions are determined by the Liouvillian $\mathcal{L}$, which gives the evolution of the density matrix, $\dot{\varrho}=\mathcal{L} \varrho$, and the averages are taken at the stationary state of this evolution, using the density matrix $\varrho_{\text {st }}$ satisfying the equation $\mathcal{L} \varrho_{\text {st }}=0$. This corresponds to measure the spectra at the latest stages of cooling, when the cavity and atom are in
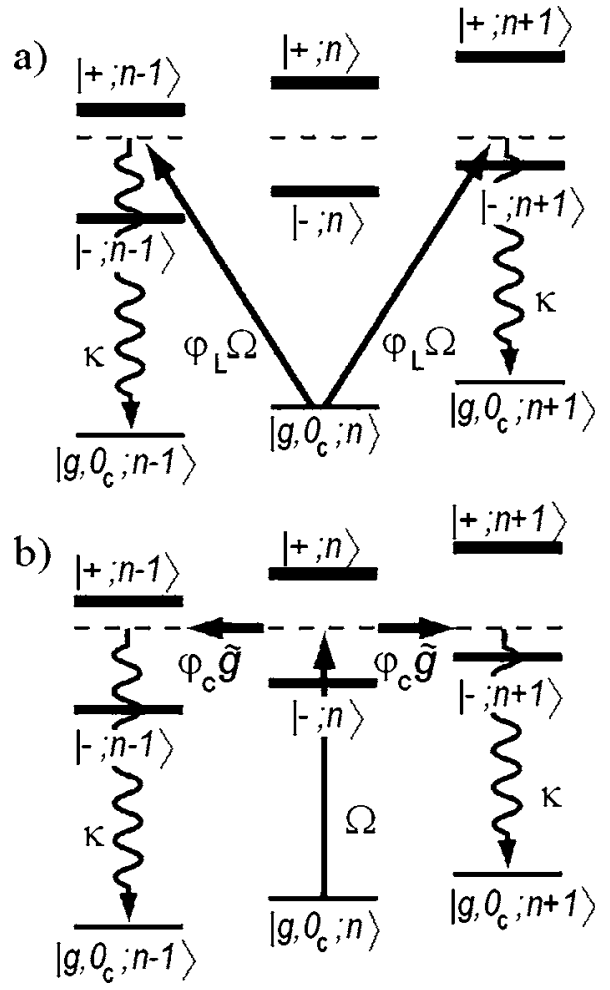

FIG. 3. Scattering processes, where the photon is emitted by cavity decay, leading to a change of the vibrational number.

the dynamical equilibrium determined by the laser and the coupling to the external environment.

The evaluation of the specific form of the spectra is made by applying the spectral decomposition of the Liouville operator $[17,33,34]$, where we use the solutions of the eigenvalue equations

$$
\begin{aligned}
& \mathcal{L} \varrho^{\lambda}=\lambda \varrho^{\lambda}, \\
& \breve{\varrho}^{\lambda} \mathcal{L}=\lambda \breve{\varrho}^{\lambda},
\end{aligned}
$$

with eigenvalues $\lambda$ and right and left eigenelements $\varrho^{\lambda}$, $\varrho^{\lambda}$, whose scalar product is defined by the trace, and fulfill the orthogonality relation $\operatorname{Tr}\left\{\check{\varrho}^{\lambda^{\prime}} \varrho^{\lambda}\right\}=\delta_{\lambda, \lambda^{\prime}}$. The Liouvillian is decomposed into the sum of projectors, $\mathcal{L}=\Sigma_{\lambda} \lambda \mathcal{P}^{\lambda}$, whose action on an arbitrary operator $X$ is defined by $\mathcal{P}^{\lambda} X$ $=\varrho^{\lambda} \operatorname{Tr}\left\{\varrho^{\lambda} X\right\}$. Accordingly, the spectra are rewritten as

$$
\mathcal{S}^{(\text {at) }}(\omega)=\operatorname{Re} \sum_{\lambda} \frac{1}{i\left(\omega-\omega_{L}\right)-\lambda} \operatorname{Tr}\left\{D^{\dagger} \mathcal{P}^{\lambda} D \varrho_{\mathrm{st}}\right\}
$$

and

$$
\mathcal{S}^{(\mathrm{cav})}(\omega)=\operatorname{Re} \sum_{\lambda} \frac{1}{i\left(\omega-\omega_{L}\right)-\lambda} \operatorname{Tr}\left\{a^{\dagger} \mathcal{P}^{\lambda} a \varrho_{\mathrm{st}}\right\}
$$

In this section we outline the procedure for evaluating these terms: in Sec. III A we define the Liouvillian $\mathcal{L}$ for the system we study, in Sec. III B we briefly discuss the steady state of the cooling dynamics, and in Sec. III C we show how Eqs. (6) and (7) are explicitly determined. 


\section{A. Liouvillian}

The system we investigate consists of an atom with mass $M$ whose center-of-mass motion is trapped by a harmonic potential of frequency $\nu$. A dipole transition connecting the ground state $|g\rangle$ to the excited state $|e\rangle$, at frequency $\omega_{0}$ and linewidth $\gamma$, is strongly coupled to a single mode of an optical cavity at frequency $\omega_{c}$. The cavity field decays at rate $\kappa$ due to the finite transmission of the mirrors. The atomic transition is driven by a laser field at frequency $\omega_{L}$, which, in combination with the resonator, cools the motion. We restrict our description of the center-of-mass motion to the $x$ axis and we indicate with $\theta_{c}$ and $\theta_{L}$ the angles that the wave vectors, $\mathbf{k}_{c}$ and $\mathbf{k}_{L}$, of the cavity mode and laser form with the $x$ axis, see Fig. 1. The coupling between atomic dipole and electromagnetic radiation is assumed to be in the LambDicke regime, when the width of the atomic wave packet is small compared with the field wavelength $2 \pi / k$, where here $\left|\mathbf{k}_{\mathbf{L}}\right| \approx\left|\mathbf{k}_{\mathbf{c}}\right|=k$. In this regime the mechanical effect of light can be studied in perturbation theory in the Lamb-Dicke parameter $\eta=k \sqrt{\hbar / 2 M \nu}$. At second order in $\eta$ we express the Liouvillian, determining the evolution of the density matrix of atom and resonator degrees of freedom, as

$$
\mathcal{L}=\mathcal{L}_{0}+\mathcal{L}_{1}+\mathcal{L}_{2}+O\left(\eta^{3}\right)
$$

where $\mathcal{L}_{j}$ is the corresponding order in the Lamb-Dicke expansion. At zeroth order in $\eta$ the external dynamics is decoupled from the dipole-cavity dynamics and

$$
\mathcal{L}_{0}=\mathcal{L}_{E}+\mathcal{L}_{I},
$$

where

$$
\mathcal{L}_{E} \varrho=-i \nu\left[b^{\dagger} b, \varrho\right]
$$

is the Liouvillian for the center-of-mass oscillator. Here $b$ and $b^{\dagger}$ are the annihilation and creation operators of a quantum of vibrational energy. Term $\mathcal{L}_{I}$ in Eq. (9) acts only on cavity and dipole degrees of freedom and it is given by

$$
\mathcal{L}_{I} \varrho=\frac{1}{i \hbar}\left[H_{\mathrm{at}}+H_{\mathrm{cav}}+H_{\mathrm{at}-\mathrm{cav}}+H_{L}, \varrho\right]+\mathcal{K} \varrho+\mathcal{L}_{s} \varrho,
$$

where

$$
\begin{aligned}
& H_{\mathrm{at}}=-\hbar \Delta \sigma^{\dagger} \sigma, \\
& H_{\mathrm{cav}}=-\hbar \delta_{c} a^{\dagger} a,
\end{aligned}
$$

with $\sigma=|g\rangle\langle e|$, and $\sigma^{\dagger}$ its adjoint, $a$, and $a^{\dagger}$ are the annihilation and creation operators of a cavity photon, $\Delta=\omega_{L}-\omega_{0}$ and $\delta_{c}=\omega_{L}-\omega_{c}$ are the detunings of the laser from the dipole and from the cavity frequency, respectively. The terms

$$
\begin{gathered}
H_{\mathrm{at}-\mathrm{cav}}=\hbar \tilde{g}\left(a^{\dagger} \sigma+a \sigma^{\dagger}\right), \\
H_{L}=\hbar \Omega\left(\sigma^{\dagger}+\sigma\right)
\end{gathered}
$$

describe the radiative couplings of the dipole with the cavity mode, at vacuum Rabi frequency $\tilde{g}$, and with the laser, at Rabi frequency $\Omega$. The coupling constant $\tilde{g}=g \cos \phi$ contains the phase $\phi$ determining the position of the trap center in the mode spatial function. The incoherent dynamics at zeroth order in $\eta$ is described by the two superoperators $\mathcal{K}$ and $\mathcal{L}_{s}$ in Eq. (8) which account for the cavity decay and dipole spontaneous emission,

$$
\begin{gathered}
\mathcal{K} \varrho=\frac{\kappa}{2}\left(2 a \varrho a^{\dagger}-a^{\dagger} a \varrho-\varrho a^{\dagger} a\right), \\
\mathcal{L}_{s} \varrho=\frac{\gamma}{2}\left(2 \sigma \varrho \sigma^{\dagger}-\sigma^{\dagger} \sigma \varrho-\varrho \sigma^{\dagger} \sigma\right) .
\end{gathered}
$$

The coupling between external and internal atomic degrees of freedom enters in the term $\mathcal{L}_{1}$ and $\mathcal{L}_{2}$ of Eq. (8). The first order Liouvillian is

$$
\mathcal{L}_{1} \varrho=-i \eta\left[\left(b^{\dagger}+b\right) V_{1}, \varrho\right]
$$

with

$$
V_{1}=\varphi_{c} V_{1 c}+\varphi_{L} V_{1 L}
$$

where

$$
\begin{gathered}
V_{1 L}=i \Omega\left(\sigma^{\dagger}-\sigma\right), \\
V_{1 c}=-\widetilde{g}\left(a \sigma^{\dagger}+a^{\dagger} \sigma\right)
\end{gathered}
$$

describe, respectively, the interaction of the atomic dipole with the laser and with the cavity field at first order in the Lamb-Dicke parameter. The mechanical effects of the laser and of the cavity field are scaled by the geometrical factors

$$
\varphi_{L}=\cos \theta_{L},
$$

$$
\varphi_{c}=\cos \theta_{c} \tan \phi .
$$

The Liouvillian at second order is

$$
\mathcal{L}_{2} \varrho=-i \eta^{2}\left[\left(b^{\dagger}+b\right)^{2}\left(\varphi_{c}^{2} V_{2 c}+\varphi_{L}^{2} V_{2 L}\right), \varrho\right]+\mathcal{L}_{2 s},
$$

where

$$
V_{2 L}=-\frac{\Omega}{2}\left(\sigma^{\dagger}+\sigma\right),
$$

$$
V_{2 c}=-\frac{\tilde{g}}{2 \tan ^{2} \phi}\left(a \sigma^{\dagger}+a^{\dagger} \sigma\right) .
$$

Superoperator $\mathcal{L}_{2 s}$ accounts for the recoil effect of photons scattered in the modes of the electromagnetic field external to the cavity,

$$
\begin{aligned}
\mathcal{L}_{2 s} \varrho= & \eta^{2} \frac{\gamma \alpha}{2} \sigma\left[2\left(b+b^{\dagger}\right) \varrho\left(b+b^{\dagger}\right)\right. \\
& \left.-\left(b+b^{\dagger}\right)^{2} \varrho-\varrho\left(b+b^{\dagger}\right)^{2}\right] \sigma^{\dagger}
\end{aligned}
$$

and $\alpha=\int_{-1}^{1} d \cos \theta \cos ^{2} \theta \mathcal{N}(\cos \theta)$ gives the angular dispersion of the atom momentum due to the spontaneous emission of photons. Finally, the dipole $D$ entering in Eq. (3) is decomposed according to $D=D_{0}+D_{1}+D_{2}$, where

$$
\begin{gathered}
D_{0}=\sigma, \\
D_{1}=-i \eta \sigma\left(b^{\dagger}+b\right) \cos \psi,
\end{gathered}
$$




$$
D_{2}=-\frac{1}{2} \eta^{2} \sigma\left(b^{\dagger}+b\right)^{2} \cos ^{2} \psi,
$$

and $\psi$ is the angle of the emitted photon with the motional axis.

\section{B. Steady state of cooling dynamics}

The averages in Eqs. (3) and (4) are taken over the stationary state of the system. This is described by the density matrix $\varrho_{\text {st }}$ satisfying $\mathcal{L} \varrho_{\text {st }}=0$, which is an eigenvalue equation for the operator $\mathcal{L}$ at eigenvalue $\lambda=0$. Thus $\varrho_{\text {st }}$ is the right eigenelement of $\mathcal{L}$ at $\lambda=0, \varrho_{\text {st }} \equiv \varrho^{0}$. At zero order in the Lamb-Dicke expansion $\varrho_{\text {st }}$ is the product of the density matrices for the internal and external degrees of freedom,

$$
\varrho_{\mathrm{st}}=\rho_{\mathrm{st}} \mu,
$$

where $\rho_{\text {st }}$ satisfy $\mathcal{L}_{I} \rho_{\text {st }}=0$, see Eq. (11), and $\mu$ is the density matrix of the external degrees of freedom, steady-state solution of the cooling equation [15]

$$
\begin{aligned}
\dot{\mu}= & \frac{\eta^{2}}{2} A_{-}\left[2 b \mu b^{\dagger}-b^{\dagger} b \mu-\mu b^{\dagger} b\right] \\
& +\frac{\eta^{2}}{2} A_{+}\left[2 b^{\dagger} \mu b-b b^{\dagger} \mu-\mu b b^{\dagger}\right],
\end{aligned}
$$

with $A_{+}<A_{-}$. In particular,

$$
\mu=\frac{1}{1+\langle n\rangle}\left(\frac{\langle n\rangle}{1+\langle n\rangle}\right)^{b^{\dagger} b},
$$

with

$$
\langle n\rangle=\operatorname{Tr}\left\{b^{\dagger} b \mu\right\}=\frac{A_{+}}{A_{-}-A_{+}},
$$

which is the average phonon number at steady state. The coefficients $A_{+}$and $A_{-}$are defined as

$$
A_{ \pm}=2 \operatorname{Re}\{s(\mp \nu)+\mathcal{D}\}
$$

with the diffusion coefficient $\mathcal{D}=\alpha \frac{\gamma}{2} \operatorname{Tr}\left\{\sigma^{\dagger} \sigma \rho_{\text {st }}\right\}$, and

$$
s(\nu)=\int_{0}^{\infty} d \tau e^{i \nu \tau} \operatorname{Tr}\left\{V_{1} e^{\mathcal{L}_{I} \tau} V_{1} \rho_{\mathrm{st}}\right\},
$$

the spectrum of fluctuation of the dipole force [32]. Using Eq. (19), Eq. (32) can be decomposed into the sum

$$
s(\nu)=\varphi_{L}^{2} s_{L}(\nu)+\varphi_{c}^{2} s_{c}(\nu)+\varphi_{L} \varphi_{c} s_{c L}(\nu),
$$

where

$$
s_{L}=-\operatorname{Tr}_{I}\left\{V_{1 L}\left(\mathcal{L}_{I}+i \nu\right)^{-1} V_{1 L} \rho_{\mathrm{st}}\right\}
$$

is the contribution of the mechanical effect due to the laser, the term

$$
s_{c}=-\operatorname{Tr}_{I}\left\{V_{1 c}\left(\mathcal{L}_{I}+i \nu\right)^{-1} V_{1 c} \rho_{\mathrm{st}}\right\}
$$

is the contribution of the mechanical effect due to the resonator, and

$$
s_{c L}=-\operatorname{Tr}_{I}\left\{V_{1 c}\left(\mathcal{L}_{I}+i \nu\right)^{-1} V_{1 L} \rho_{\mathrm{st}}\right\}-\operatorname{Tr}_{I}\left\{V_{1 L}\left(\mathcal{L}_{I}+i \nu\right)^{-1} V_{1 c} \rho_{\mathrm{st}}\right\}
$$

is the contribution due to correlations between the mechanical effects of the laser and resonator. Depending on the geometry of the setup and on the value of the laser and cavity parameter, one term can be dominant over the others.

In the rest of the paper we assume a weak laser driving the atom. This allows us to restrict the description of the coupled dipole-cavity dynamics to the lowest internal levels, namely the ground state $\left|g, 0_{c}\right\rangle$, where $0_{c}$ indicates the vacuum state of the cavity field, and the first two excited states $\left|g, 1_{c}\right\rangle$ and $\left|e, 0_{c}\right\rangle$ with one excitation in the dipolecavity system. Checks of consistency show that this approximation is reliable for the parameter regimes we considered. Nevertheless, the center of mass motion is treated with no approximation, and thus is a harmonic oscillator, with its infinite ladder of equispaced levels.

\section{Evaluation of spectra}

In the regime of validity of the Lamb-Dicke expansion the spectra in Eqs. (6) and (7) are evaluated by determining the eigenvalues and eigenvectors of $\mathcal{L}$ in perturbation theory in $\eta$, by solving iteratively the secular equations (5) at the same order in the perturbative expansion.

At zero order in $\eta$ internal and external degrees of freedom are decoupled, see Eq. (9). Therefore the eigenvalues of $\mathcal{L}_{0}$ are $\lambda_{0}=\lambda_{\mathrm{I}}+\lambda_{\mathrm{E}}$, and the eigenelements $\varrho_{0}^{\lambda}=\rho^{\lambda_{\mathrm{I}}} \mu^{\lambda_{\mathrm{E}}}$ where $\mathcal{L}_{I} \rho^{\lambda_{\mathrm{I}}}=\lambda_{\mathrm{I}} \rho^{\lambda_{\mathrm{I}}}$ and $\mathcal{L}_{\mathrm{E}} \mu^{\lambda_{\mathrm{E}}}=\lambda_{\mathrm{E}} \mu^{\lambda_{\mathrm{E}}}$. The corresponding projector is

$$
\mathcal{P}_{0}^{\lambda}=\mathcal{P}^{\lambda} \mathcal{U}^{\lambda} \text { E, }
$$

whereby their action on the operator $X$ is defined as

$$
\begin{gathered}
\mathcal{P}^{\lambda_{\mathrm{I}}} X=\rho^{\lambda_{\mathrm{I}}} \operatorname{Tr}_{\mathrm{I}}\left\{\breve{\rho}^{\lambda_{\mathrm{I}}} X\right\}, \\
\mathcal{U}^{\lambda_{\mathrm{E}}} X=\mu^{\lambda_{\mathrm{E}}} \operatorname{Tr}_{\mathrm{E}}\left\{\breve{\mu}^{\lambda_{\mathrm{E}}} X\right\},
\end{gathered}
$$

and $\operatorname{Tr}_{\mathrm{I}}\left(\operatorname{Tr}_{\mathrm{E}}\right)$ denotes the trace over the internal (external) degrees of freedom. In the limit of a motionless atom the power spectra are characterized by the eigenvalues of $\mathcal{L}_{I}$. The effect of the atomic motion enters with the appearance of features at frequencies $\lambda_{I}+\lambda_{E}$, with $\lambda_{\mathrm{E}}=i \ell \nu$ and $\ell$ $=0, \pm 1, \pm 2, \ldots$. At zero order in $\eta$ each eigenspace at $\lambda_{\mathrm{E}}$ is infinitely degenerate, and the corresponding left and right eigenelements are, for instance, $\check{\mu}_{n}^{\ell}=|n+\ell\rangle\left\langle n\left|, \mu_{n}^{\ell}=\right| n\right\rangle\langle n$ $+\ell \mid$. These eigenelements constitute a complete and orthonormal basis over the eigenspace at this eigenvalue. In particular, the projector over the eigenspace at $\lambda_{\mathrm{E}}=i \ell \nu$ is defined on an operator $X$ as

$$
\mathcal{U}^{\lambda} \mathrm{E}^{=i \ell}{ }^{\nu} X=\sum_{n} \mu_{n}^{\ell} \operatorname{Tr}_{\mathrm{E}}\left\{\mu_{n}^{\ell} X\right\}=\sum_{n}|n\rangle\langle n|X| n+\ell\rangle\langle n+\ell|,
$$

where $\operatorname{Tr}_{\mathrm{E}}$ denotes the trace over the external degrees of freedom.

At higher orders in the expansion in $\eta$, internal and external degrees of freedom are coupled, and the degeneracy of 
the subspaces at eigenvalue $\lambda_{\mathrm{E}}$ is lifted [35]. We do not report explicit results for higher order expressions of $\lambda, \varrho^{\lambda}$, and $\mathcal{P}^{\lambda}$ here, since - although the physical systems are intrinsically different-the technique for gaining these relevant expressions is presented in detail in Refs. [16,17]. Using the expansion in $\eta$, the spectra in Eqs. (6) and (7) are decomposed as

$$
\mathcal{S}^{(j)}(\omega)=\mathcal{S}_{0}^{(j)}(\omega)+\mathcal{S}_{2}^{(j)}(\omega)+O\left(\eta^{3}\right),
$$

where the term $\mathcal{S}_{\alpha}^{(j)}(\omega)$ is at order $\alpha$ in the expansion in $\eta$. In zero order, the spectra are

$$
\begin{gathered}
\mathcal{S}_{0}^{\text {(at) }}(\omega)=\operatorname{Re} \sum_{\lambda_{\mathrm{I}}} \frac{1}{i\left(\omega-\omega_{L}\right)-\lambda_{\mathrm{I}}} \operatorname{Tr}_{\mathrm{I}}\left\{D_{0}^{\dagger} \mathcal{P}^{\lambda_{\mathrm{I}}} D_{0} \rho_{\mathrm{st}}\right\}, \\
\mathcal{S}_{0}^{\text {(cav) }}(\omega)=\operatorname{Re} \sum_{\lambda_{\mathrm{I}}} \frac{1}{i\left(\omega-\omega_{L}\right)-\lambda_{\mathrm{I}}} \operatorname{Tr}_{\mathrm{I}}\left\{a^{\dagger} \mathcal{P}^{\lambda_{\mathrm{I}}} a \rho_{\mathrm{st}}\right\}
\end{gathered}
$$

and represent the spectral signal when the atom is at rest. The first nonvanishing correction is of second order,

$$
\mathcal{S}_{2}^{(j)}(\omega)=\operatorname{Re} \sum_{\lambda} \frac{1}{i\left(\omega-\omega_{L}\right)-\lambda} F^{(j)}(\lambda)
$$

and it describes the spectral features due to mechanical effects of the photon-atom interaction. We introduced the weighting functions at the eigenvalue $\lambda$,

$$
\begin{gathered}
F^{\text {(at) }}(\lambda)=\sum_{\alpha+\beta+\gamma+\delta=2} \operatorname{Tr}\left\{D_{\alpha}^{\dagger} \mathcal{P}_{\beta}^{\lambda} D_{\gamma} \varrho_{\delta}^{\text {st }}\right\}, \\
F^{\text {(cav) }}(\lambda)=\sum_{\alpha+\beta=2} \operatorname{Tr}\left\{a^{\dagger} \mathcal{P}_{\alpha}^{\lambda} a \varrho_{\beta}^{\text {st }}\right\},
\end{gathered}
$$

where $\alpha, \beta, \gamma$, and $\delta$ denote the order in the Lamb-Dicke expansion of the corresponding quantities. The resulting expressions are quite lengthy and we abstain from reporting them here. Their plots are presented in the next section for some relevant parameter regimes.

\section{RESULTS}

In this section we discuss the spectra of emission of atomic fluorescence $\mathcal{S}^{(\text {at })}$ and of cavity transmitted field $\mathcal{S}^{\text {(cav) }}$, as evaluated from Eq. (37). They are obtained when the width of the atomic transition $\gamma$ is much larger than the trap frequency $\nu$ and $\nu \gg \kappa$, at steady state of the cooling dynamics discussed in $[14,15]$. We remark that, in order to extract the power spectrum, the curves presented in this section must be multiplied with the corresponding factor $\chi^{(j)}$, see Eq. (2).

\section{A. Spectrum for cavity assisted sideband cooling}

We first analyze the case in which the atomic motion is at steady state of cavity sideband cooling $[14,15]$. Here, the laser is set on the red sideband transition of the cavity mode, $\delta_{c} \sim-\nu$, and is far off resonance from the atomic dipole transition, $|\Delta| \gg \gamma$. The atom-cavity states which are mostly involved in the cooling dynamics are $\left|g, 0_{c}\right\rangle$ and $\left|g, 1_{c}\right\rangle$, while
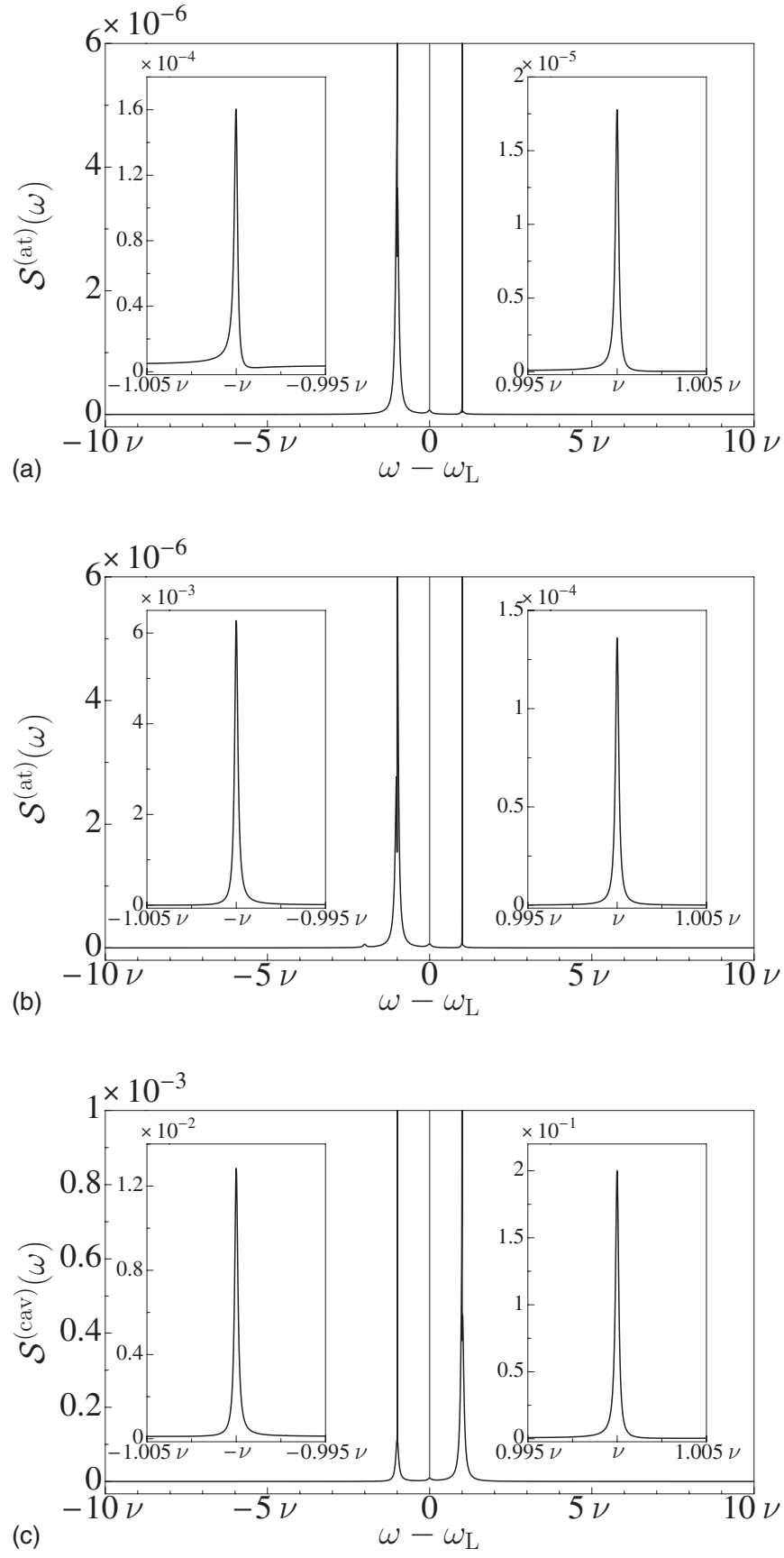

FIG. 4. Spectra of emission at steady state of cavity sideband cooling. (a) and (b) report the spectrum of resonance fluorescence spectrum for the detector angles $\psi=0$ and $\psi=\pi$, respectively, (c) is the spectrum at the cavity output. The plots show the $\delta$-like elastic peak at $\omega_{\mathrm{L}}$, the narrow sidebands of the elastic peak at $\omega_{\mathrm{L}} \pm \nu$, and small signals of the inelastic spectrum which are due to the mechanical coupling. The spectral components resulting from mechanical effects depend on the detector position. The signals at $\omega$ $=\omega_{L} \pm \nu$, including the sidebands of the elastic peak, are magnified in the insets. The parameters are $\delta_{c}=-\nu, \Delta=500 \nu, \Omega=5 \nu, \widetilde{g}=7 \nu$, $\gamma=10 \nu, \kappa=0.1 \nu, \eta=0.05, \phi=\pi / 4$, and $\varphi_{\mathrm{c}}=\varphi_{\mathrm{L}}=1 / \sqrt{2}$.

the excited state $\left|e, 0_{c}\right\rangle$ is almost empty at all stages. The corresponding spectra of emission are displayed in Fig. 4 in the frequency region around the laser frequency. We identify features, which are commonly observed in the resonance 
fluorescence of atoms, undergoing sideband cooling in free space, namely the elastic peak at the laser frequency and the sidebands of the elastic peak, which are due to the harmonic motion of the atom and whose relative height depends on the angle of emission [30]. In fact, the atom-cavity system in this case constitutes an effective two-level transition between the states $\left|g, 0_{c}\right\rangle$ and $\left|g, 1_{c}\right\rangle$. The spectra of resonance fluorescence corresponding to the angles $\psi=0, \pi$, where the effect of photon recoil on the atomic motion is maximum, are plotted in Figs. 4(a) and 4(b). Differing to the case of sideband cooling in free space the spectrum of resonance fluorescence exhibits an enhancement at frequency $\omega_{L}-\nu$, corresponding to a heating transition, while an enhancement at the cooling transition, at $\omega_{L}+\nu$, is visible in the spectrum at the cavity output, see Fig. 4(c). In fact, the photons which cool the atom are mostly lost by cavity decay, while correspondingly the photons which heat the motion are far off resonance from the cavity mode, being $\kappa \ll \nu$, and are hence mostly emitted by spontaneous decay. We checked that the intensity at the upper and lower sidebands, integrated over the solid angle, are equal, consistent with the fact that the system is at steady state of the cooling dynamics. Finally, we note that weak sidebands of the inelastic contribution at $\omega_{L}-\nu$ are visible in the spectrum of resonance fluorescence, see Figs. 4(a) and 4(b). They are located at $\omega_{\mathrm{L}}$ and $\omega_{\mathrm{L}}-2 \nu$ and are due to the atomic motion, see [17].

\section{B. Suppression of inelastic spectrum}

More striking features, which differ dramatically from the spectrum of resonance fluorescence of a dipole in free space, are encountered when the cooling processes are enhanced by an interference effect between the driving laser and the field scattered into the resonator. This phenomenon occurs when the laser is resonant with the cavity mode, $\delta_{c}=0$, and for ideal resonators $(\kappa=0)$ it leads to perfect suppression of atomic excitation for a fixed and pointlike particle [36]. It is recovered in high finesse resonators, provided that the cooperativity is sufficiently large. In this case, most of the light is emitted through the cavity mirrors [37].

When the finite size of the atomic wave packet is considered, the gradient of the electromagnetic field over the atomic wave packet gives rise to absorption, which can be shaped in order to achieve efficient cooling [15]. In this regime, the excitation appearing in Fig. 2(a) is suppressed, due to the destructive interference between the absorption paths $\left|g, 0_{c}, n\right\rangle \rightarrow| \pm, n\rangle$ which do not change the motional excitation. The corresponding spectrum of resonance fluorescence is displayed in Fig. 5(a). Due to the interference effect, the elastic peak and the inelastic spectrum contributions scale with $\kappa^{2}$ and $\eta^{4}$, respectively, and are very small. The spectrum is dominated by the sidebands of the elastic peak which are symmetric, and whose form is independent of the angle of emission. In fact, due to the destructive interference effect, the angle dependent diffusive processes depicted in Fig. 2(a) are suppressed. This behavior has been also encountered in the spectrum of resonance fluorescence of a three-level atom in $\Lambda$ configuration driven under the electromagnetic induced transparency condition [16]. There, photon scattering at zero
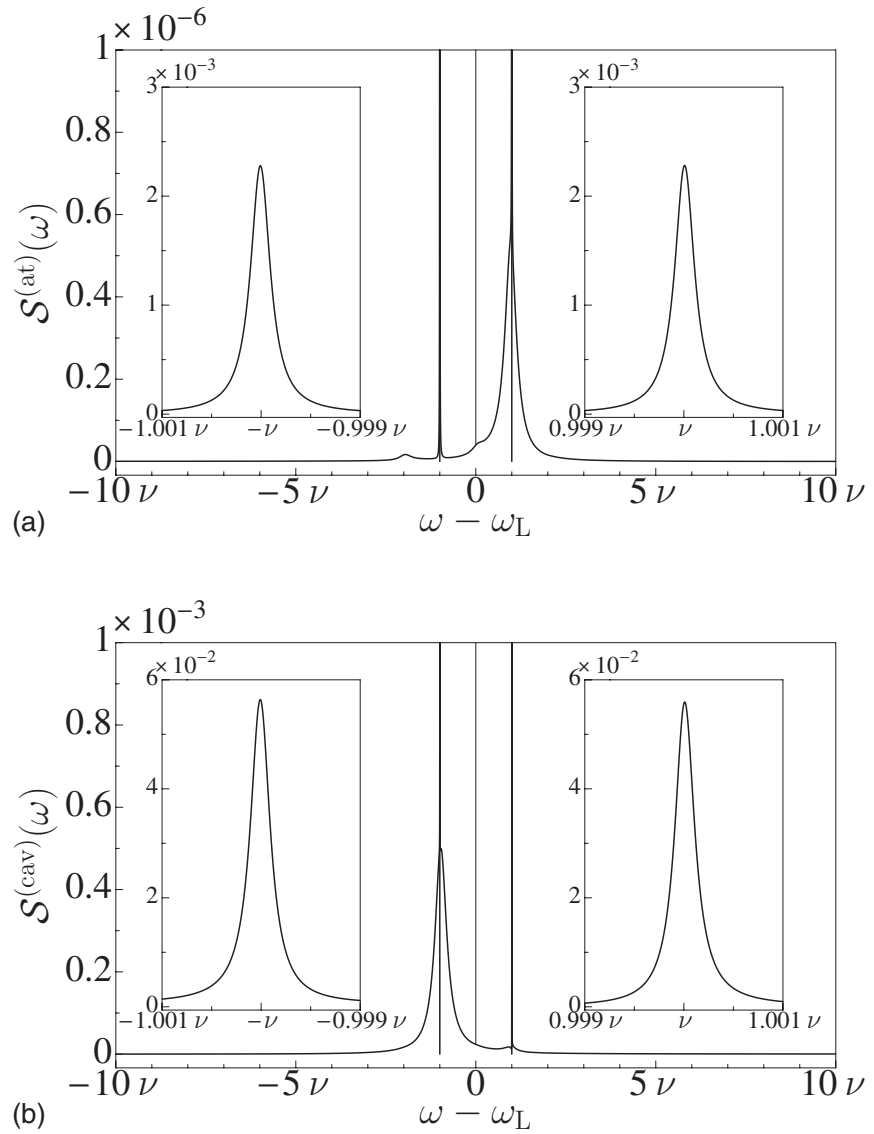

FIG. 5. (a) Spectrum of resonance fluorescence and (b) spectrum of the cavity output when the atomic motion is cooled by a scheme based on destructive interference between the laser and cavity fields, which tends to suppress atomic fluorescence [15]. The spectra are dominated by the motional sidebands at $\omega_{\mathrm{L}} \pm \nu$. The broader signal in (b) stems from inelastically scattered light. In the insets the motional sidebands are magnified. The parameters are $\delta_{c}=0, \quad \Delta=48 \nu, \Omega=0.5 \nu, \quad \tilde{g}=7 \nu, \quad \gamma=10 \nu, \quad \kappa=0.01 \nu, \quad \eta=0.05, \quad \phi$ $=\pi / 4$, and $\varphi_{\mathrm{c}}=\varphi_{\mathrm{L}}=1 / \sqrt{2}$.

order in the Lamb-Dicke expansion was suppressed due to quantum interference in the electronic excitations. Here, it is suppressed due to interference between the laser and cavity field at the atomic position [37].

Figure 5(b) displays the spectrum at the cavity output. Here, elastic peak and inelastic spectral signals scale with $\kappa$ and are visible. The inelastic component is centered at the lower sideband frequency, showing that the photons which are emitted by the resonator heat the atomic motion. The sidebands of the elastic peak have predominant Lorentzian character and equal height. However, a weak dispersive behavior of the sidebands is visible in Fig. 5(b), which is due to the interference between the scattering processes depicted in Fig. 3.

\section{Suppression of lower sideband signal}

We now focus on the spectra obtained when cooling is based on the suppression of the heating transition [15]. This behavior stems from interference between the mechanical ef- 

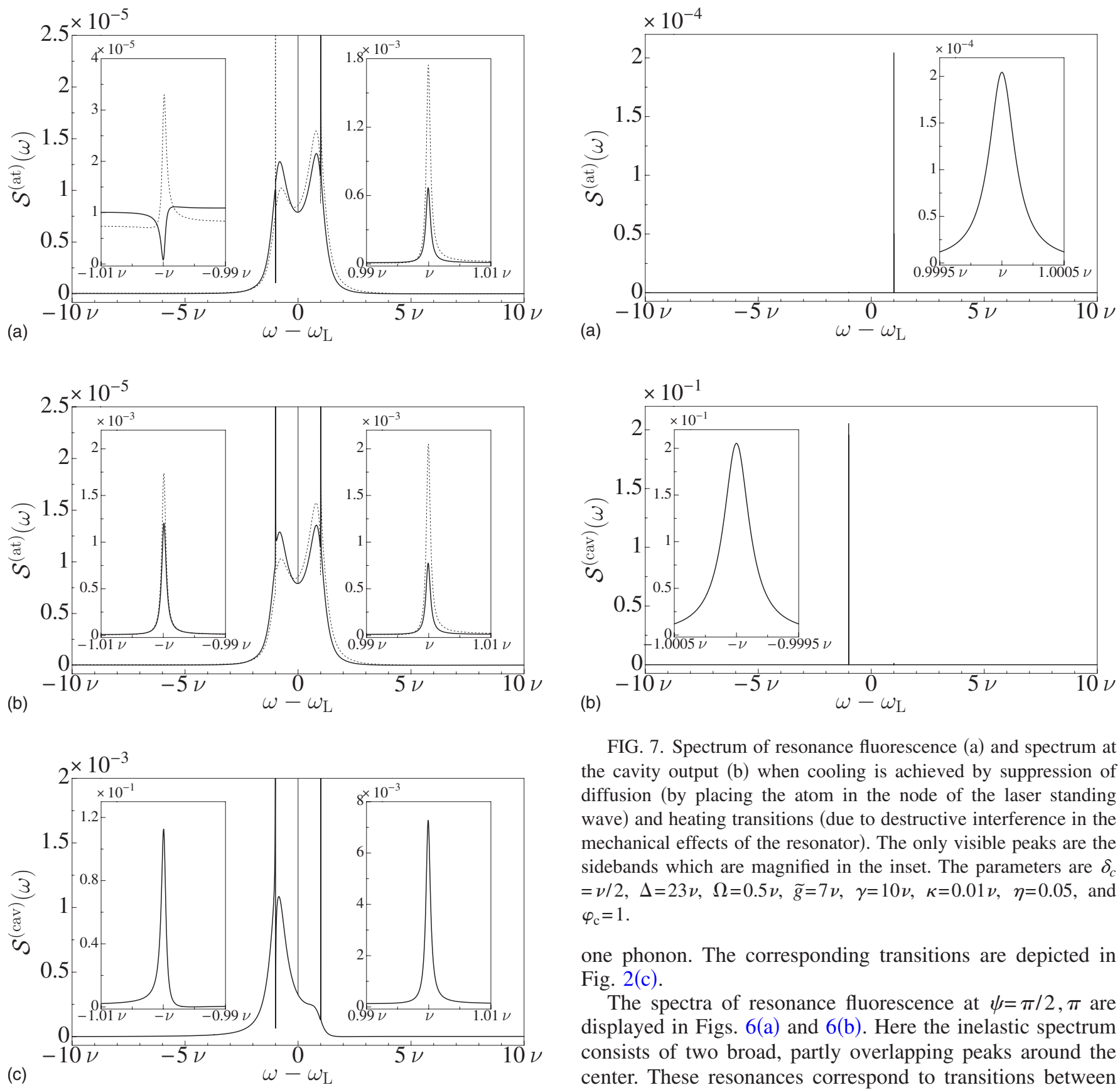

FIG. 7. Spectrum of resonance fluorescence (a) and spectrum at the cavity output (b) when cooling is achieved by suppression of diffusion (by placing the atom in the node of the laser standing wave) and heating transitions (due to destructive interference in the mechanical effects of the resonator). The only visible peaks are the sidebands which are magnified in the inset. The parameters are $\delta_{c}$ $=\nu / 2, \Delta=23 \nu, \Omega=0.5 \nu, \tilde{g}=7 \nu, \gamma=10 \nu, \kappa=0.01 \nu, \eta=0.05$, and $\varphi_{\mathrm{c}}=1$.

one phonon. The corresponding transitions are depicted in Fig. 2(c).

The spectra of resonance fluorescence at $\psi=\pi / 2, \pi$ are displayed in Figs. 6(a) and 6(b). Here the inelastic spectrum consists of two broad, partly overlapping peaks around the center. These resonances correspond to transitions between the dressed states of the cavity and the ground state at zero order in the Lamb-Dicke expansion, see [25]. Other inelastic contributions to the spectrum are very weak and lie outside the frequency range displayed in the figures. The motional sidebands of the elastic peak are asymmetric and depend on the angle of emission. This can be understood in terms of interfering scattering processes between the mechanical effects of the spontaneously emitted photon and of the cavity field, which are sketched in Figs. 2(c) and 2(a), respectively. The lower sideband vanishes at the angle $\psi=\pi / 2$, see Fig. 6(a), when the direction of emission is orthogonal to the motion and there is no contribution of spontaneous emission: In this case, the quantum interference effect suppressing the heating transitions is clearly evident in the spectrum, leading to the disappearance of the signal at $\omega_{L}-\nu$. The dashed line corresponds to the signal when the resonator is unstable, 
with $\kappa=0.1 \nu$. In this case the suppression of the heating sideband is not perfect, still this signal is orders of magnitude smaller than the cooling sideband at $\omega_{\mathrm{L}}+\nu$. Figure 6(b) shows the spectrum of resonance fluorescence when the recoil of the emitted photon is maximum $(\psi=\pi)$, and thus this process interferes with the heating photon, giving a dispersivelike signal at $\omega_{L}-\nu$.

The corresponding spectrum at the cavity output is displayed in Fig. 6(c) for $\kappa=0.01 \nu$. The left motional sideband at $\omega_{\mathrm{L}}-\nu$ shows a dispersivelike behavior. The total intensity at this frequency is larger than the upper sideband signal. This is understood, as the interference process leading to suppression of the heating transition is taking place between the scattering amplitudes leading to spontaneous emission. Correspondingly, the heating photons are mostly emitted by cavity decay and give rise to this signal.

\section{Monochromatic spectrum of resonance fluorescence}

Finally, we consider the spectra of emission when the atom is driven by a standing-wave laser with the trap center placed at one of its nodes, and the cavity parameters are such that the heating transitions are suppressed by destructive interference. In this configuration, the contributions to the spectra at zero order in the Lamb-Dicke expansion defined in Eqs. (38) and (39) are suppressed because of the vanishing field at the node of the standing wave, and the resulting spectra of scattered light are only due to mechanical effects of the photon-atom interaction. In Figs. 7(a) and 7(b) one can observe that the only relevant signals are the sidebands of the elastic peak, which have Lorentzian form. In particular, due to the interference effect suppressing the heating transition, the spectrum of resonance fluorescence is only constituted by the cooling sideband, while the spectrum at the cavity output is only constituted by the heating motional sideband. Correspondingly, the photons emitted by a heating transition are only detected at the cavity output.

\section{CONCLUSION}

We have analyzed the spectrum of resonance fluorescence and the spectrum at the cavity output of a cavity cooled atom. In general, the two spectra contain complementary information about the scattering processes determining the atomic dynamics. In certain parameter regimes features may appear only in one spectrum as the result of quantum interference between transitions induced by the laser and cavity fields.

These results provide further insight into the coupled dynamics of atoms and resonators and can be used for implementing quantum feedback schemes, in the spirit of the proposals of Refs. [38-40] and of the experimental realization in [13].

\section{ACKNOWLEDGMENTS}

One of the authors (M.B.) thanks the group d'Optica at the Universitat Autonoma de Barcelona for hospitality during completion of this work. Support by the European Commission (EMALI, MRTN-CT-2006-035369; SCALA, Contract No. 015714), by the Spanish Ministerio de Educación y Ciencia (Consolider Ingenio 2010 QOIT, CSD2006-00019; QLIQS, FIS2005-08257), and by the Alexander-vonHumboldt Foundation are acknowledged.
[1] P. W. H. Pinkse, T. Fisher, P. Maunz, and G. Rempe, Nature (London) 404, 365 (2000).

[2] C. J. Hood, T. W. Lynn, A. C. Doherty, and H. J. Kimble, Science 287, 1447 (2000).

[3] M. A. Wilson, P. Bushev, J. Eschner, F. Schmidt-Kaler, C. Becher, R. Blatt, and U. Dorner, Phys. Rev. Lett. 91, 213602 (2003).

[4] P. Bushev, A. Wilson, J. Eschner, C. Raab, F. Schmidt-Kaler, C. Becher, and R. Blatt, Phys. Rev. Lett. 92, 223602 (2004).

[5] H. W. Chan, A. T. Black, and V. Vuletic, Phys. Rev. Lett. 90, 063003 (2003); A. T. Black, H. W. Chan, and V. Vuletic, ibid. 91, 203001 (2003).

[6] D. Kruse, C. von Cube, C. Zimmermann, and Ph. W. Courteille, Phys. Rev. Lett. 91, 183601 (2003); C. von Cube, S. Slama, D. Kruse, C. Zimmermann, Ph. W. Courteille, G. R. M. Robb, N. Piovella, and R. Bonifacio, ibid. 93, 083601 (2004).

[7] B. Nagorny, Th. Elsässer, and A. Hemmerich, Phys. Rev. Lett. 91, 153003 (2003).

[8] P. Maunz, T. Puppe, I. Schuster, N. Syassen, P. W. H. Pinkse, and G. Rempe, Nature (London) 428, 50 (2004).

[9] J. McKeever, J. R. Buck, A. D. Boozer, A. Kuzmich, H. C. Nägerl, D. M. Stamper-Kurn, and H. J. Kimble, Phys. Rev.
Lett. 90, 133602 (2003).

[10] P. Domokos and H. Ritsch, J. Opt. Soc. Am. B 20, 1098 (2003).

[11] P. Horak, H. Ritsch, T. Fischer, P. Maunz, T. Puppe, P. W. H. Pinkse, and G. Rempe, Phys. Rev. Lett. 88, 043601 (2002).

[12] T. Fischer, P. Maunz, P. W. H. Pinkse, T. Puppe, and G. Rempe, Phys. Rev. Lett. 88, 163002 (2002).

[13] P. Bushev, D. Rotter, A. Wilson, F. Dubin, C. Becher, J. Eschner, R. Blatt, V. Steixner, P. Rabl, and P. Zoller, Phys. Rev. Lett. 96, 043003 (2006).

[14] V. Vuletic, H. W. Chan, and A. T. Black, Phys. Rev. A 64, 033405 (2001).

[15] S. Zippilli and G. Morigi, Phys. Rev. Lett. 95, 143001 (2005); Phys. Rev. A 72, 053408 (2005).

[16] M. Bienert, W. Merkel, and G. Morigi, Phys. Rev. A 69, 013405 (2004); see also M. Bienert, Ph.D. thesis, University of Ulm, 2004 (Mensch \& Buch Verlag, Berlin, 2004).

[17] M. Bienert, W. Merkel, and G. Morigi, Phys. Rev. A 73, 033402 (2006).

[18] J. J. Sanchez-Mondragon, N. B. Narozhny, and J. H. Eberly, Phys. Rev. Lett. 51, 550 (1983).

[19] H. J. Carmichael, Phys. Rev. Lett. 55, 2790 (1985).

[20] R. Bonifacio and L. A. Lugiato, Phys. Rev. Lett. 40, 1023 
(1978); Phys. Rev. A 18, 1129 (1978).

[21] P. D. Drummond and D. F. Walls, Phys. Rev. A 23, 2563 (1981); H. J. Carmichael, D. F. Walls, P. D. Drummond, and S. S. Hassan, Phys. Rev. A 27, 3112 (1983).

[22] H. J. Carmichael, R. J. Brecha, M. G. Raizen, H. J. Kimble, and P. R. Rice, Phys. Rev. A 40, 5516 (1989); C. M. Savage, Phys. Rev. Lett. 63, 1376 (1989).

[23] M. Xiao, H. J. Kimble, and H. J. Carmichael, Phys. Rev. A 35, 3832 (1987); W. Ren, J. D. Cresser, and H. J. Carmichael, ibid. 46, 7162 (1992); W. Ren and H. J. Carmichael, ibid. 51, 752 (1995).

[24] H. J. Kimble, in Cavity Quantum Electrodynamics, edited by P. R. Berman (Academic Press, New York, 1994), p. 203.

[25] H. Freedhoff and T. Quang, J. Opt. Soc. Am. B 10, 1337 (1993); Phys. Rev. Lett. 72, 474 (1994).

[26] J. Leach and P. R. Rice, Phys. Rev. Lett. 93, 103601 (2004).

[27] P. Domokos and H. Ritsch, Phys. Rev. Lett. 89, 253003 (2002).

[28] T. Salzburger and H. Ritsch, Phys. Rev. Lett. 93, 063002 (2004); T. Salzburger, P. Domokos, and H. Ritsch, Phys. Rev. A 72, 033805 (2005).

[29] M. Lindberg, Phys. Rev. A 34, 3178 (1986).

[30] J. I. Cirac, R. Blatt, A. S. Parkins, and P. Zoller, Phys. Rev. A 48, 2169 (1993).

[31] Ch. Raab, J. Eschner, J. Bolle, H. Oberst, F. Schmidt-Kaler, and R. Blatt, Phys. Rev. Lett. 85, 538 (2000).

[32] J. I. Cirac, R. Blatt, P. Zoller, and W. D. Phillips, Phys. Rev. A 46, 2668 (1992).

[33] H. J. Briegel and B.-G. Englert, Phys. Rev. A 47, 3311 (1993); for a review, see B.-G. Englert and G. Morigi, in Coherent Evolution in Noisy Environments, Lecture Notes in Physics Vol. 611, edited by A. Buchleitner and K. Hornberger (Springer-Verlag, Berlin, 2002), p. 55, and references therein.

[34] S. M. Barnett and S. Stenholm, J. Mod. Opt. 47, 2869 (2000); see also S. M. Barnett and S. Stenholm, Phys. Rev. A 64, 033808 (2001), and references therein.

[35] J. Javanainen, M. Lindberg, and S. Stenholm, J. Opt. Soc. Am. B 1, 111 (1984); M. Lindberg and S. Stenholm, J. Phys. B 17, 3375 (1985).

[36] P. M. Alsing, D. A. Cardimona, and H. J. Carmichael, Phys. Rev. A 45, 1793 (1992).

[37] S. Zippilli, G. Morigi, and H. Ritsch, Phys. Rev. Lett. 93, 123002 (2004); Eur. Phys. J. D 31, 507 (2004).

[38] S. Mancini, D. Vitali, and P. Tombesi, Phys. Rev. Lett. 80, 688 (1998); Phys. Rev. A 61, 053404 (2000).

[39] D. A. Steck, K. Jacobs, H. Mabuchi, T. Bhattacharya, and S. Habib, Phys. Rev. Lett. 92, 223004 (2004).

[40] P. Rabl, V. Steixner, and P. Zoller, Phys. Rev. A 72, 043823 (2005); V. Steixner, P. Rabl, and P. Zoller, ibid. 72, 043826 (2005). 